\title{
AVALIAÇÃO DA DENSIDADE MINERAL ÓSSEA EM PACIENTES COM DOENÇA INFLAMATÓRIA INTESTINAL
}

\author{
Fabiana Lígia LORA ${ }^{1}$, Heda M. B. AMARANTE ${ }^{1}$, Júlio C. PISANI ${ }^{1}$, \\ Victória V. C. BORBA ${ }^{2}$, Carolina A. M. KULAK ${ }^{2}$ e Eliane R. CARMES ${ }^{3}$
}

RESUMO - Racional - Pacientes com doença inflamatória intestinal têm maior prevalência de redução da densidade mineral óssea em comparação às pessoas saudáveis. Objetivo - Avaliar a densidade mineral óssea em uma população de pacientes com doença inflamatória intestinal. Métodos - Noventa pacientes de 20 a 50 anos de idade, do ambulatório de doença inflamatória intestinal do Serviço de Gastroenterologia do Hospital de Clínicas da Universidade Federal do Paraná, Curitiba, PR, foram selecionados para avaliação. Desses, 76 completaram todas as etapas de avaliação. A densitometria foi realizada da coluna lombar e fềmur direito em aparelho Hologyc QDR 1000/W. Resultados - Os pacientes com doença inflamatória intestinal tiveram significativa redução da densidade mineral óssea, avaliada por massa óssea em números absolutos $\left(\mathrm{g} / \mathrm{cm}^{2}\right)$, em todas as regiões avaliadas, colo do fềmur, fềmur total e coluna lombar. As variáveis analisadas como, índice de atividade de doença, uso de corticóide, cirurgias prévias, índice de massa corpórea e falta de atividade física, não demonstraram correlação com a massa óssea, ou seja, não influenciaram os resultados da densidade mineral óssea no grupo estudado de doentes com doença inflamatória intestinal. Conclusão - Densidade mineral óssea reduzida foi encontrada nos pacientes com doença inflamatória intestinal do Ambulatório de Doença Inflamatória Intestinal do Serviço de Gastroenterologia do Hospital de Clínicas da Universidade Federal do Paraná, mais pronunciadamente nos pacientes com doença de Crohn, semelhante ao descrito na literatura. Nenhuma das variáveis analisadas mostrou correlação com a densidade mineral óssea.

DESCRITORES - Densidade óssea. Enteropatias inflamatórias. Osteoporose.

\section{INTRODUÇÃO}

Pacientes com doença inflamatória intestinal (DII) têm maior prevalência de redução da densidade mineral óssea (DMO) em comparação com as pessoas saudáveis. A osteopenia é encontrada em taxas que variam de $40 \%$ a $75 \%$ entre pacientes com DII, enquanto a freqüência de osteoporose varia de $2 \%$ a $30 \%$, dependendo do grupo estudado ${ }^{(1,5,6,9,10,11,24)}$.

A diminuição da DMO ocasiona aumento da fragilidade óssea e do risco de fraturas, o que pode elevar a morbidade e diminuir a qualidade de vida neste grupo de pacientes ${ }^{(17,19)}$.

Os mecanismos responsáveis pela perda óssea ainda são pouco compreendidos. Entre os diversos fatores sugeridos como envolvidos na patogênese da osteopenia nos pacientes com DII, estão a má absorção de cálcio e vitamina $\mathrm{D}$ causada pela inflamação da mucosa intestinal ou ressecção intestinal, o uso de corticóides, a redução da atividade física e a atividade inflamatória da própria doença $^{(4,12,14)}$.

A retocolite ulcerativa inespecífica (RCUI) e a doença de Crohn (DC) são moléstias imunomediadas, caracterizadas por uma resposta inflamatória crônica. Embora a causa da DII não esteja, ainda, claramente definida, a ativação de células $\mathrm{T}$ e a liberação de citocinas com atividades imunorreguladoras e pró-inflamatórias, como a interleucina1 , interleucina- 6 e fator de necrose tumoral alfa parecem contribuir para a gênese da inflamaçãa ${ }^{(18)}$. Independentemente da atividade inflamatória sistêmica, estas citocinas têm também função importante na estimulação parácrina do desenvolvimento dos osteoclastos e na regulação da reabsorção óssea, o que pode contribuir para a alteração do metabolismo ósseo nos pacientes com DII ${ }^{(13)}$.

O objetivo deste estudo foi avaliar a densidade mineral óssea na população de pacientes com DII atendida no Serviço de Gastroenterologia do Hospital de Clínicas da Universidade Federal do Paraná (UFPR), Curitiba, PR, e compará-la a um grupo controle de pessoas saudáveis.

\section{CASUÍSTICA E MÉTODOS}

\section{Pacientes}

Noventa de 190 pacientes entre 20 e 50 anos de idade, acompanhados no ambulatório de DII do Serviço de Gastroenterologia do Hospital de Clínicas da UFPR, foram selecionados para este estudo. Destes, 14 pacientes

${ }^{1}$ Disciplinas de Gastroenterologia, ${ }^{2}$ Endocrinologia e ${ }^{3}$ Epidemiologia da Faculdade de Medicina da Universidade Federal do Paraná, Curitiba, PR

Endereço para correspondência: Dra. Fabiana Lígia Lora - Rua Pasteur, 486 - apt. 901 - Batel - 80250-080 - Curitiba, PR. E-mail: fabianall@uol.com.br 
não completaram a etapa de realização da densitometria óssea. Dos 76 pacientes que completaram a avaliação, 39 tinham DC, com média de idade de 32 anos, sendo 21 homens. Trinta e sete pacientes tinham RCUI, com média de idade de 36 anos, sendo 25 mulheres. O diagnóstico e extensão da doença foram confirmados por endoscopia, radiologia e histologia antes do início do estudo. Foram excluídas todas as mulheres após a menopausa, aquelas com ciclos menstruais irregulares ou em uso de terapia estrogênica. Foram excluídos, também, pacientes sabidamente acometidos de outras doenças que causem alteração no metabolismo ósseo, tais como hepatopatia crônica, hipogonadismo, hipoparatireoidismo, hiper ou hipotireoidismo, artrite reumatóide, lúpus eritematoso sistêmico, insuficiência renal crônica, doença pulmonar obstrutiva crônica grave e outras doenças que causem diarréia e má absorção que não a DII.

Os critérios clínicos avaliados foram o índice de atividade e a duração da doença, o segmento intestinal comprometido e sua extensão, os segmentos ressecados nos pacientes já submetidos a cirurgias, o índice de massa corporal, a prática de atividade física e inquérito dietético e de hábitos de vida. $\mathrm{Na}$ avaliação do índice de atividade da doença, foram utilizados os índices de TRULOVE e WITTS ${ }^{(25)}$ para RCUI e de BEST ${ }^{(7)}$ para DC. Ambos baseiam-se em questionários de sinais e sintomas e exame físico, tais como número e consistência das evacuações, presença de dor abdominal e sintomas extra-intestinais ou de complicações, avaliação de qualidade de vida, entre outros critérios clínicos.

Calculou-se a dose cumulativa de corticóide do último ano e dose média diária, expressas em gramas de prednisona, e o tempo, em meses, sem uso do corticóide.

Quarenta controles saudáveis que nunca haviam se submetido a cirurgia intestinal, com média de idade de 34 anos foram estudados. Nenhum dos controles utilizava ou havia utilizado corticóide, diuréticos, colestiramina ou heparina.

Consentimento informado escrito foi obtido dos pacientes e controles antes do estudo.

\section{Densitometria óssea}

A densitometria óssea foi realizada no Serviço de Endocrinologia do Hospital de Clínicas da UFPR no mesmo dia da coleta dos exames laboratoriais e da coleta das informações clínicas. O exame foi medido por "dual-energy x-ray absortiometry" (Hologyc QDR 1000/W) da coluna lombar (L1-L2-L3-L4, vista ântero-posterior) e fêmur direito. Os resultados foram expressos em $\mathrm{g} / \mathrm{cm}^{2}$ e desvios padrões dos valores normais, que foram supridos pelos níveis normais do Hologic.

Osteopenia foi definida como diminuição na densidade mineral óssea maior que 1 , desvio padrão abaixo da média da população jovem normal e osteoporose como densidade maior que 2,5, desvios padrões abaixo desta média.

\section{Análise estatística}

Foi realizada análise descritiva dos dados através de tabelas, quadros e gráficos. Para a comprovação do objetivo do estudo foram utilizados a análise da variância (ANOVA), o coeficiente de correlação de Pearson e o teste paramétrico $t$ de Student e os não-paramétricos Mann-Whitney, comparação entre duas proporções (através do software Primer of Biostatistics), Qui-quadrado e exato de Fisher (pelo software Epi-Info). O nível de significância (probabilidade de significância) adotado foi menor que $5 \%(P<0,05)$.

\section{RESULTADOS}

O total de 90 pacientes do Ambulatório de Doenças Inflamatórias Intestinais do Hospital de Clínicas da UFPR foi avaliado para este estudo, entre os meses de maio e novembro de 2003. Destes, 14 não completaram a etapa da densitometria óssea, sendo excluídos do mesmo. Dos 76 pacientes que completaram a avaliação, 37 tinham RCUI e 39 doença de Crohn. Quarenta pessoas saudáveis foram avaliadas para o grupo controle. A amostra estudada era homogênea em relação à idade, sexo, raça, tabagismo, etilismo, índice de massa corpórea e atividade física.

A Tabela 1 mostra as conclusões das densitometrias ósseas nos diferentes grupos do estudo.

TABELA 1 - Conclusão da densitometria óssea nos grupos de estudo

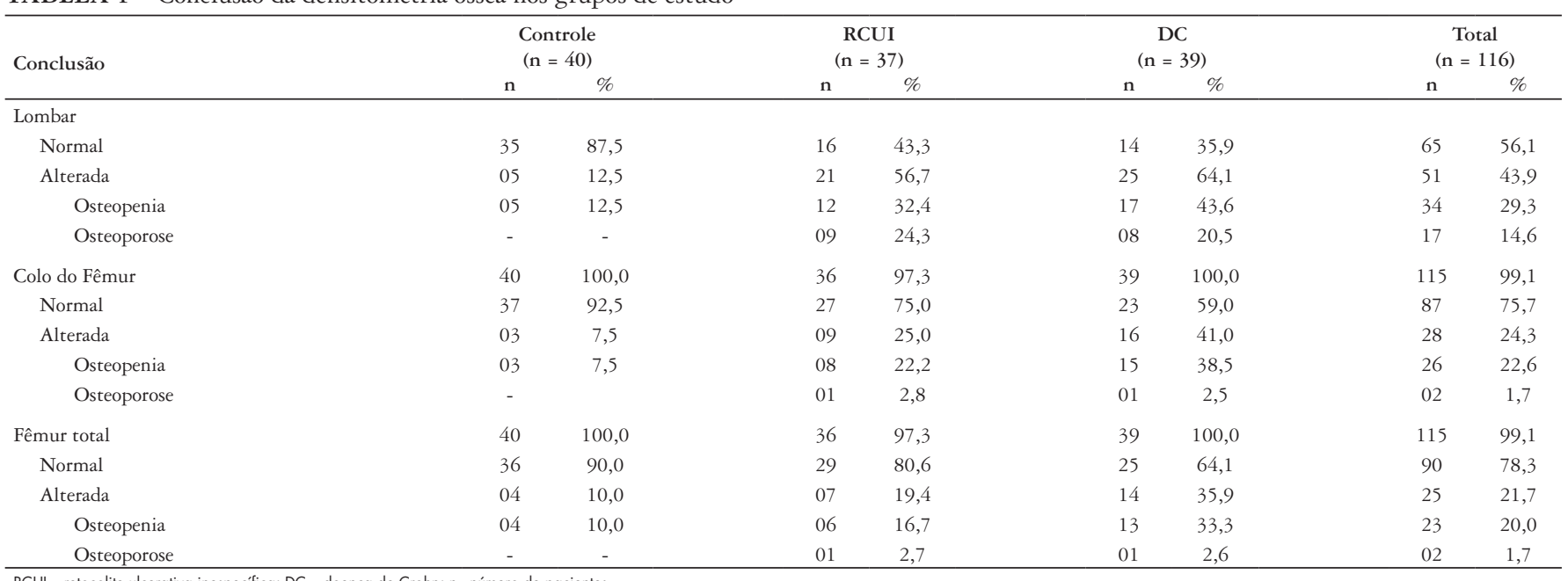

RCUI - retocolite ulcerativa inespecífica; DC - doença de Crohn; $n$ - número de pacientes 
A densidade mineral óssea, avaliada por massa óssea em números absolutos $\left(\mathrm{gramas} / \mathrm{cm}^{2}\right)$, apresentou-se com valores menores nos portadores de DII, em comparação ao grupo controle, sendo esta diferença significativa $(P<0,05)$ (Figuras 1 e 2).

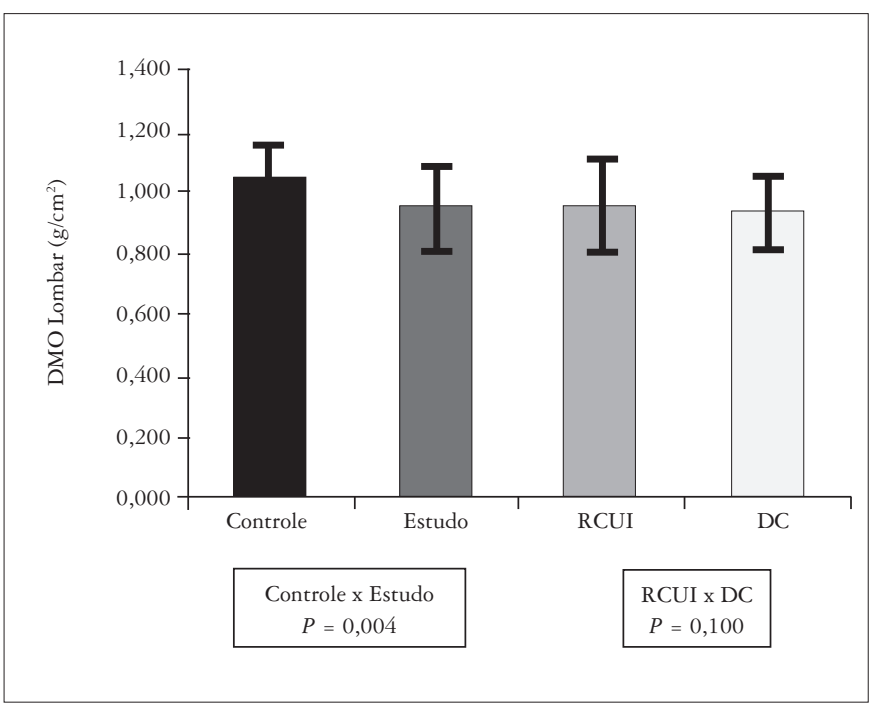

FIGURA 1 - DMO da coluna lombar nos grupos de estudo RCUI - retocolite ulcerativa inespecífica; DC - doença de Crohn; DMO - densidade mineral óssea; Grupo de estudo $=$ RCUI + DC

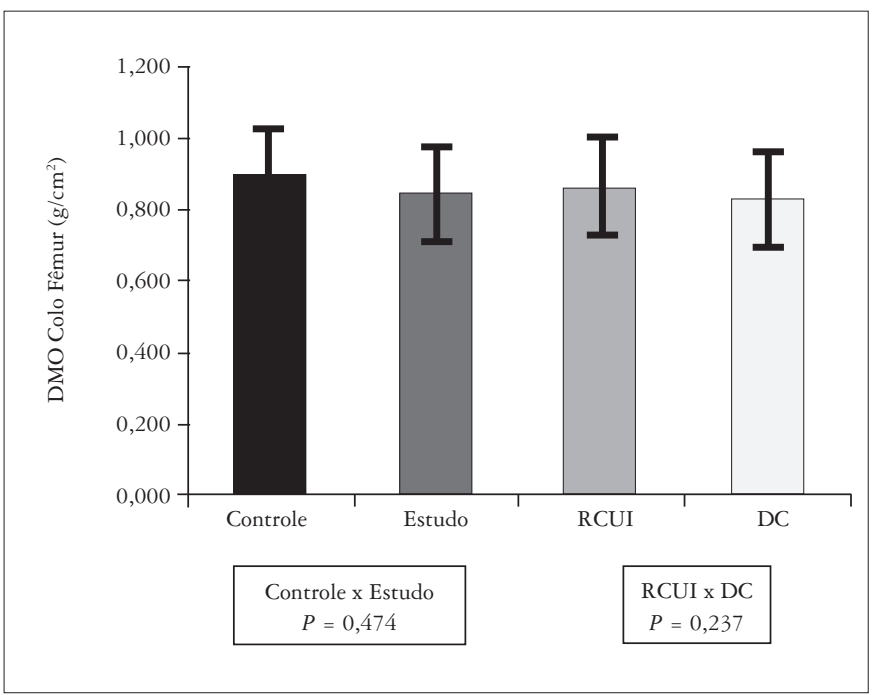

FIGURA 2 - DMO do colo do fêmur nos grupos de estudo RCUI - retocolite ulcerativa inespecífica; DC - doença de Crohn; DMO - densidade mineral óssea; Grupo de estudo $=$ RCUI + DC

O grupo de DC foi o que apresentou os menores índices de massa óssea, com diferença estatística significativa, em todos os segmentos ósseos avaliados (DMO lombar, colo do fêmur e fêmur total).
Na comparação entre os grupos de DC e RCUI, não houve diferença importante entre eles em nenhuma das três regiões ósseas avaliadas, coluna lombar $(P=0,474)$, colo do fềmur $(P=0,237)$ e fêmur total $(P=0,191)$.

Quando comparados os diagnósticos finais da DMO definidos pelo $\mathrm{Z}$ escore, também se observou que os pacientes com DII têm DMOs freqüentemente mais alteradas que o grupo controle, sendo a maior parte das alterações compreendida por osteopenia (Figura 3).

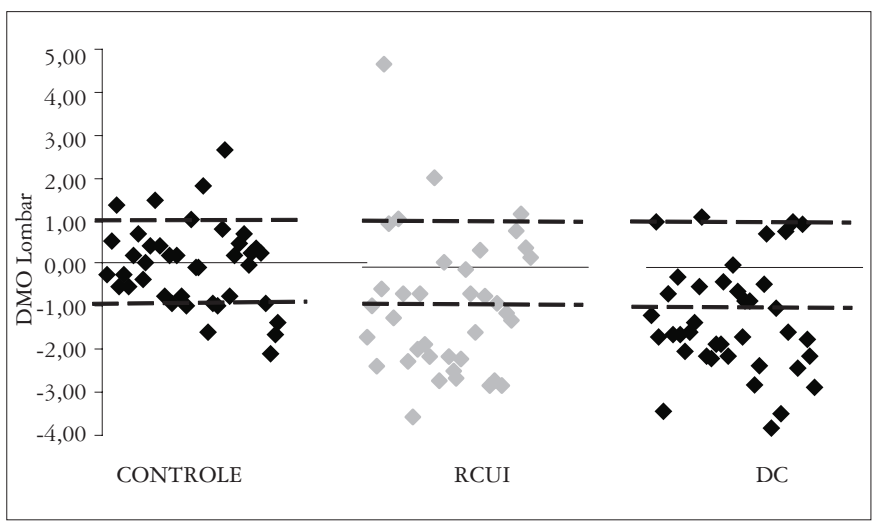

FIGURA $3-z$ escore da DMO lombar por grupo de estudo

RCUI - retocolite ulcerativa inespecífica; DC - doença de Crohn; DMO - densidade mineral óssea

Neste estudo as correlações pelo teste de exato de Fischer entre o índice de atividade da doença e a densitometria óssea foram muito baixas e sem diferença significativa, demonstrando ausência de associação entre estas variáveis na amostra avaliada.

$\mathrm{Na}$ correlação entre a dose média diária de corticóide no último ano (mg/dia) e a densitometria óssea, para a DMO lombar houve baixa correlação, sem diferença significativa $(r=0,2218$; $P=0,2304)$. O mesmo ocorreu para a DMO do colo do fêmur. No entanto, observou-se probabilidade limítrofe, indicando tendência nos pacientes com as menores doses de corticóide terem também menores índices de DMO (dentro da normalidade, entre 0,800 e 1,000).

DMO fêmur total apresentou correlação regular com diferença significativa $(\mathrm{r}=0,4459 ; P=0,0119)$, ou seja, doses de corticóide mais baixas tendem a ter DMO menores (dentro da normalidade, entre 0,800 e 1,000 ).

$\mathrm{Na}$ correlação dos dados de cirurgia prévia (colectomia e enterectomia) e densitometria óssea, não foi observada diferença entre os pacientes que realizaram em relação aos que não realizaram cirurgia.

Também não houve diferença estatisticamente significativa entre as densitometrias dos pacientes que realizavam atividade física regular (três ou mais dias por semana) e daqueles que não a faziam $(P>0,1)$.

O índice de massa corporal (IMC) foi a variável que mostrou ter correlação com a DMO, porém não significativa, dentro dos limites da normalidade nos dois grupos de pacientes, em todos os segmentos ósseos avaliados com $P=0,0100$ a $P=0.289$. As relações da DMO lombar com o IMC nos grupos com RCUI e DC estão representadas na Tabela 2. 
TABELA 2 - Correlação entre o índice de massa corporal e a densitometria óssea, no grupo de estudo

\begin{tabular}{lcc}
\hline Dados & Correlação $(\mathbf{r})$ & Valor de $P(1)$ \\
\hline RCUI & & \\
• DMO lombar & 0,3871 & 0,0180 \\
- DMO colo fêmur & 0,4237 & 0,0100 \\
- DMO fêmur total & 0,4200 & 0,0108 \\
DC & & \\
- DMO lombar & 0,1771 & 0,2809 \\
- DMO colo fêmur & 0,3500 & 0,0290 \\
- DMO fêmur total & 0,3530 & 0,0275 \\
\hline 1 - coeficiente de correlação de Pearson; r - correlação; RCUI - retocolite ulcerativa inespecífica; \\
DC - doença de Crohn; DMO - densidade mineral óssea
\end{tabular}

\section{DISCUSSÃO}

Pacientes com DII têm prevalência maior de alterações da $\mathrm{DMO}$ em relação à população normal, como relatado em muitos artigos, que demonstram prevalências de $30 \%$ a $75 \%$ de diminuição da densidade óssea no grupo de portadores destas doenças ${ }^{(1,5,9,22)}$. A perda óssea é o maior fator de risco para fraturas osteoporóticas, agregando importante morbidade à doença previamente existente ${ }^{(17)}$. Há um ano, pela primeira vez, isso foi demonstrado por KLAUS et al..$^{(19)}$, que encontrou uma ou mais fraturas vertebrais em $21,8 \%$ dos pacientes com DC que apresentavam diminuição da DM.

Este estudo transversal confirma os relatos prévios de alteração da densidade mineral óssea nos pacientes com $\mathrm{DII}^{(2,12,21)}$. A população estudada tem os valores da DMO significativamente menores $(P<0,05)$ em relação aos indivíduos saudáveis, em todas as regiões ósseas avaliadas (coluna lombar, colo de fêmur e fêmur total).

$\mathrm{O}$ osso trabecular, especialmente as vértebras, é geralmente mais afetado que o osso cortical, embora ambos possam estar $\operatorname{alterados}^{(11)}$. Esta diferença ficou demonstrada em ambos os grupos de pacientes avaliados neste estudo. Na RCUI a coluna lombar apresentou alteração em 56,7\% dos casos contra 19,4\% de alteração encontrada no fêmur. Na DC esta diferença foi de $64,1 \%$ contra $35,9 \%$, respectivamente, sempre com predomínio da osteopenia em relação à osteoporose.

Estes resultados corroboram com estudos comparativos que demonstraram que os pacientes com DC têm a maior redução na densidade mineral óssea, quando comparados aos demais grupos, controles e RCUI ${ }^{(14)}$. A diferença da DMO com relação ao grupo controle foi significativa, o que não ocorreu na comparação com os portadores de RCUI, diferentemente do relatado em outras populações avaliadas ${ }^{(4,15)}$. Diferença essa estatisticamente não significativa nos segmentos ósseos avaliados $(P=0,191-0,474)$.

Muitas pesquisas têm demonstrado que o processo inflamatório no trato intestinal pode estar diretamente relacionado às alterações no metabolismo ósseo em pacientes com $\mathrm{DII}^{(1)}$. Mediadores e citocinas liberadas pelas células inflamatórias intestinais, como IL-1, IL-6 e fator de necrose tumoral alfa, que se sabe estarem elevados em pacientes com DII, estimulam a atividade dos osteoclastos, inibem a atividade dos osteoblastos e podem contribuir para o desequilíbrio das citocinas reguladoras do metabolismo ósseo ${ }^{(13,18,23)}$. Assim, o nível de atividade inflamatória, representado pelo índice de atividade da doença, apresentaria correlação com as alterações da DMO. Porém, como em levantamento anterior ${ }^{(8)}$, este trabalho não demonstrou correlação importante entre fase de atividade da doença e alterações ósseas. No entanto, é necessário considerar que a maioria dos pacientes estava em fase de remissão ou com leve atividade de doença. Poucos pacientes apresentavam índice de atividade moderado e nenhum deles tinha atividade intensa, conforme os índices aplicados. Talvez outros parâmetros de atividade inflamatória e do metabolismo ósseo, como a dosagem dos mediadores inflamatórios e marcadores bioquímicos de reabsorção óssea, apresentem maior correlação com as anormalidades do metabolismo ósseo ${ }^{(1,2,13)}$.

$\mathrm{O}$ efeito deletério do corticóide no metabolismo ósseo é bastante conhecido, porém é controversa a relevância das doses e tempo de uso do corticóide exógeno como fator causal na osteoporose $^{(20,26,27)}$. Uma meta-análise realizada em 2002, com 66 estudos de avaliação de densitometria óssea e 23 estudos de avaliação de fraturas, ambos relacionados ao corticóide, demonstrou evidências de que a alteração óssea induzida pelo corticóide e suas conseqüências são substancialmente reduzidas após a suspensão da terapia. Esta redução de risco acontece significativamente após 1 ano da parada do uso do corticóide ${ }^{(28)}$. Em função disto, no presente estudo, optou-se pela quantificação da dose média diária do último ano $(\mathrm{mg} / \mathrm{dia})$ para avaliar a intervenção do emprego do corticóide na amostra estudada.

Estudos prévios realizados em pacientes com DII são bastante conflitantes quanto à alteração da massa óssea determinada pelo corticóide ${ }^{(1,9,15,24)}$. Neste trabalho comparou-se a DMO nos grupos de pacientes com e sem uso de corticóide e não se demonstrou diferença significativa, o que contraria relatos anteriores de correlações negativas significantes entre corticóide e massa óssea nos pacientes com DII ${ }^{(3,14,24)}$. Importante observação foi que 4 dos 76 pacientes avaliados, que nunca usaram corticóide, apresentaram alteração na DMO, sendo que 1 deles, com 23 anos de idade, apresentava osteoporose na quarta vértebra lombar.

A falta de atividade física regular e cirurgias de ressecção intestinal prévias, não influenciaram a massa óssea medida nos pacientes com DII, em concordância com outros estudos ${ }^{(3,14)}$.

A baixa correlação do IMC com a DMO discorda de estudos prévios que mostraram ser este um dos principais preditores da diminuição da massa óssea nos portadores de DII ${ }^{(16)}$.

\section{CONCLUSÃO}

Encontrou-se densidade mineral óssea reduzida nos pacientes com DII do Ambulatório da Gastroenterologia do Hospital de Clínicas da UFPR, mais acentuada nos pacientes com DC, semelhante ao descrito na literatura. O IMC, o uso de corticóide, a atividade física, o índice de atividade doença e a ressecção intestinal não apresentaram correlação importante com a DMO. 
Lora FL, Amarante HMB, Pisani JC, Borba VVC, Kulak CAM, Carmes ER. Bone mineral density evaluation in inflammatory bowel disease patients. Arq Gastroenterol 42(4):201-5.

ABSTRACT - Background - Inflammatory bowel disease patients have shown greater reduction of the bone mineral density compared to healthy people. Aim - To evaluate the bone mineral density in a population of patients with inflammatory bowel disease. Methods - Ninety patients from 20 to 50 years old, of the Inflammatory Bowel Disease Ambulatory of the Gastroenterology Service of the Clinics Hospital, Curitiba, PR, Brazil, were selected for the evaluation. From those, 76 completed all the stages of the evaluation. The densitometry was made from lumbar column and right femur with a dual-energy x-ray absortiometry (Hologyc QDR 1000/W) device. Results - The inflammatory bowel disease patients had a significant reduction of the bone mineral density in all the evaluated parts, femur neck, total femur and lumbar column. The analysed variables, disease activity index, usage of corticoids, the lack of physical activities, the index body mass and previous surgeries did not have influence in the results. Conclusion - Reduced bone mineral density was founded in inflammatory bowel disease patients of the Clinics Hospital, mainly in the Crohn's disease patients, as described in literature. None analyzed variables had significant correlation to the bone mineral density.

HEADINGS - Bone density. Inflammatory bowel diseases. Osteoporosis.

\section{REFERÊNCIAS BIBLIOGRÁFICAS}

1. Abitbol V, Rooux C, Chaussade S, Guillemant S, Kolta S, Dougados M, Couturier D, Amor B. Metabolic bone assessment in patients with inflammatory bowel disease. Gastroenterology 1995; 108:417-22

2. Adachi JD, Rostom A. Metabolic bone disease in adults with inflammatory bowel disease. Inflamm Bowel Dis 1999;5:200-11.

3. Andreassen H, Hylander E, Rix M. Gender, age, and body weight are the major predictive factors for bone mineral density in Crohn's disease: a case-control crosssectional study of 113 patients. Am J Gastroenterol 1999;94:824-8.

4. Ardizzone S, Bollani S, Bettica P, Bevilacqua M, Molteni P, Bianch Porro G. Altered bone metabolism in inflammatory bowel disease: there is a difference between Crohn's disease and ulcerative colitis. J Intern Med 2000;247:63-70.

5. Bernstein $\mathrm{CN}$. Calcium and bone issues in inflammatory bowel disease. Gastroenterol Int 1997;10:71-7.

6. Bernstein CN, Leslie WD, Taback SP. Bone density in a population-based cohort of premenopausal adult women with early onset inflammatory bowel disease. Am J Gastroenterol 2003;98:1094-100.

7. Best WR, Becktel JM, Singleton JW. Rederived values of eight coefficients of Crohn's disease activity index. Gastroenterology 1979;77:843-6.

8. Bischoff SC, Herrmannn A, Göke M, Manns MP, von zur Mühlen A, Brabant G. Altered metabolism in inflammatory bowel disease. Am J Gastroenterol 1997;92:1157-63.

9. Bjarnason I, Macpherson A, Mackintosh C, Buxton-Thomas M, Forgacs I, Monitz C. Reduced bone density in patients with inflammatory bowel disease. Gut 1997; 40:228-33

10. Compston JE, Judd D, Crawley EO, Evans WD, Evans C, Church HA, Reid EM, Rhodes J. Osteoporosis in patients with inflammatory bowel disease. Gut 1987;28:410-5.

11. Compston JE. Osteoporosis, corticosteroids and inflammatory bowel disease. Aliment Pharmacol Ther 1995;9:237-50.

12. Dinca M, Fries W, Luisetto G, Peccolo F, Bottega F, Leone L, Naccarato R, Martin A. Evolution of osteopenia in inflammatory bowel disease. Am J Gastroenterol 1999;94:1292-7.

13. Epstein FH. Bone marrow, cytokines, and bone remodeling. N Engl J Med 1995;332:305-11.

14. Habtezion A, Silverberg MS, Parkes R, Mikolainis S, Steinhart H. Risk factors for low bone density in Crohn's disease. Inflamm Bowel Dis 2002;8:87-92.

15. Jahnsen J, Falch JA, Aadland E, Mowinckel P. Bone mineral density is reduced in patient with Crohn's disease but not in patients with ulcerative colitis: a population based study. Gut 1997;40:313-9.
16. Jong DJ, Corstens FH, Mannaerts L, van Rossum LG, Naber AH. Corticosteroidinduced osteoporosis: does it occur in patient with Crohn's disease? Am J Gastroenterol 2002;97:2011-5.

17. Kanis JA. Diagnosis of osteoporosis and assessment of fracture risk. Lancet 2002;359:1929-36.

18. Karlinger K, Györke T, Makö E, Mester A, Tarján A. The epidemiology and the pathogenesis of inflammatory bowel disease. Eur J Radiol 2000;35:154-67.

19. Klaus J, Armbrecht G, Steinkamp M, Brückel J, Rieber A, Adler G, Reinshagen M, Felsenberg D, von Tipitz C. High prevalence of osteoporotic vertebral fractures in patients with Crohn's disease. Gut 2002;51:654-8.

20. Reid DM. Corticosteroid-induced osteoporosis. In: Smith R, editor. Osteoporosis London: Royal College of Physicians of London; 1990. p.99-117.

21. Schoon EJ, Bolk BM, Geerling BJ, Russel MG, Stockbrügger RW, Brummer RJ. Bone mineral density in patients with recently diagnosed inflammatory bowel disease. Gastroenterology 2000;119:1203-8

22. Schoon EJ, van Nunen AB, Wouters RS, Stockbrugger RW, Russel MG. Osteopenia and osteoporosis in Crohn's disease: prevalence in a Dutch population-based cohort Scand J Gastroenterol 2000; Suppl 232:43-7.

23. Schulte CM, Dignass AU, Goebell H, Röher HD, Schulte KM. Genetic factors determine extent of bone loss in inflammatory bowel disease. Gastroenterology 2000;119:909-20.

24. Silvennoinen JA, Kattunen TJ, Niemelä SE, Manelius JJ, Lehtola JK. A controlled study of bone mineral density in patients with inflammatory bowel disease. Gut 1995;37:71-6.

25. Truelove SC, Witts LJ. Cortisone in ulcerative colitis. Final report on a therapeutic trial. Br Med J 1985;2:117-9.

26. van Staa TP, Leufkens HG, Abenhaim L, Zhang B, Cooper C. Oral corticosteroids and fracture risk: relationship to daily and cumulative doses. Rheumatology 2000;39:1383-9.

27. van Staa TP, Leufkens HG, Abenhaim L, Zhang B, Cooper C. Use of oral corticosteroids and risk of fractures. J Bone Miner Res 2000;15:993-1000

28. van Staa TP, Leufkens HGM, Cooper C. The epidemiology of corticosteroids-induced osteoporosis: a meta-analysis. Osteoporos Int 2002;13:777-87. 